\title{
Effectiveness of a modified version of the applied relaxation technique in treatment of perimenopausal and postmenopausal symptoms
}

This article was published in the following Dove Press journal:

International Journal of Women's Health

4 November 2013

Number of times this article has been viewed

\author{
Suprawita Saensak ${ }^{1,2}$ \\ Teraporn Vutyavanich ${ }^{3}$ \\ Woraluk Somboonporn ${ }^{4}$ \\ Manit Srisurapanont ${ }^{5}$ \\ 'Academic Department, Faculty of \\ Medicine, Mahasarakham University, \\ Maha Sarakham, ${ }^{2}$ Department of \\ Community Medicine, Faculty of \\ Medicine, Chiang Mai University, \\ Chiang Mai, ${ }^{3}$ Department of \\ Obstetrics and Gynecology, \\ Chiang Mai University, Chiang Mai, \\ ${ }^{4}$ Department of Obstetrics and \\ Gynecology, KhonKaen University, \\ KhonKaen, ${ }^{5}$ Department of Psychiatry, \\ Faculty of Medicine, Chiang Mai \\ University, Chiang Mai, Thailand
}

Correspondence: Suprawita Saensak Faculty of Medicine, Maha Sarakham University, Nakornsawan Road, 44000 Maha Sarakham, Thailand Tel +0660437 I 2992

Fax +06 60 437| 299|

Email psaensak@gmail.com
Background: Awareness of the risks associated with hormone therapy for menopausal symptoms has sparked a global decline in this treatment. Alternative treatments to relieve menopausal symptoms are therefore required. The applied relaxation (AR) technique has proven to be successful for symptom amelioration, but requires participation in 12 weekly classes. The purpose of this study was to determine the effectiveness of a modified relaxation version (MR) of AR for treatment of hot flashes, night sweats, and sleep disturbances.

Methods: We conducted a12-week, randomized, parallel, open-label, controlled trial in perimenopausal and postmenopausal women visiting the menopausal clinic. Participants were randomly assigned to an MR or AR group. The MR group $(n=36)$ received a single session of (MR) training and the AR group $(n=35)$ received conventional 12-week training. Participants were instructed to practice the techniques daily at home for 12 weeks. The main outcome was the measure on the severity scale and frequency of hot flashes, night sweats, and sleep disturbances.

Results: All participants completed the study. Total severity scores in both groups decreased after 12 weeks, but there was no difference between the groups $(P=0.93)$. The severity score for hot flashes in the MR group decreased more than in the AR group $(P=0.02)$. The severity scores for night sweats and sleep disturbances decreased in both groups. The frequency of hot flashes, night sweats, and sleep disturbances were also decreased in both groups.

Conclusion: A shorter, modified version of the AR was equally effective or slightly better than the conventional AR for the relief of hot flashes, night sweats, and sleep disturbances in perimenopausal and postmenopausal women. Recommendations for future research include confirmatory studies and trials with larger samples.

Keywords: alternative treatments, applied relaxation, menopausal symptoms, hot flashes

\section{Introduction}

Most women in menopause report at least one symptom, such as hot flashes, night sweats, or sleep disturbances. ${ }^{1}$ The prevalence of these symptoms varies widely and is likely to be influenced by a range of factors, including climate, diet, lifestyle, women's roles and attitudes regarding aging, and the end of reproductive capacity. ${ }^{1,2}$ Hot flashes are the most common symptom, reported in $30 \%-50 \%$ of perimenopausal women, and are a result of cutaneous vasodilatation. ${ }^{1,3,4}$ This symptom usually begins as an ascending flash of the upper body, starting from the thorax, and results in a sensation of warmth. The vasodilatation also causes a decrease in body core temperature, resulting in a sensation of cold that often elicits shivering. Hot flashes often occur at night (night sweats) and disrupt normal sleep patterns. ${ }^{5}$ Some studies have indicated 
that this symptom may peak during the later stages of the menopausal transition when women are missing periods, ${ }^{6,7}$ and some recent studies have shown that these symptoms can continue for longer than previously thought. ${ }^{8}$ In contrast, sleep disorders seem to increase in a linear fashion over the menopausal transition and the postmenopausal period. ${ }^{6}$

The perimenopausal period is defined as the period immediately before menopause when the endocrine, biological, and clinical manifestations begin to appear, and includes the first year after menopause begins. The postmenopausal period dates from the final menstrual period, regardless of whether menopause was induced or spontaneous. Nearly $10 \%$ of women in the US undergoing perimenopausal or menopausal changes seek medical advice from health care providers. ${ }^{9}$ Estrogen therapy is a well established and effective treatment for menopausal symptoms. ${ }^{10}$ However, following publication of the Women's Health Initiative study, ${ }^{11}$ the prescription and use of hormone therapy has declined globally due to awareness of the risks associated with hormone therapy. ${ }^{12}$ Therefore, many symptomatic women are seeking alternative treatments. ${ }^{13-15}$ Relaxation techniques, such as paced respiration (a type of slow, deep breathing that uses the diaphragm to do the work of inhaling and exhaling), muscle relaxation or a combination of both (applied relaxation [AR]) are well known behavioral treatments for menopausal symptoms. ${ }^{16}$ The goal of the AR technique is to achieve rapid relaxation by correct breathing in situations with bothersome symptoms, ie, hot flashes. ${ }^{17}$ These techniques effectively attenuate the frequency ${ }^{18}$ and intensity ${ }^{18}$ of hot flashes, thereby minimizing discomfort and sleep disturbances. ${ }^{12}$

AR, the most commonly used relaxation method for treating menopausal complaints, shows promise for alleviating vasomotor and other symptoms. ${ }^{19}$ However, the AR technique involves intensive training over 12 consecutive weeks. Each session lasts for 60 minutes, and individuals are asked to practice at home for at least 15-20 minutes per day. More than $25 \%$ of individuals drop out of the training course as a result of this time commitment. MR training is a modification of the AR technique that shortens the training time and emphasizes home practice. This shorter version requires participants to attend only one 60-minute session, which is expected to reduce the dropout rate. The results of this preliminary study of the modified relaxation (MR) technique at the Mahasarakham Provincial Hospital resulted in all participants completing the program and reporting dramatic improvement in their vasomotor symptoms.

The present randomized controlled trial compared the effects of traditional AR with those of a one-session
MR intervention for the relief of menopausal symptoms in Thai women. Outcome variables included change in intensity and frequency of hot flashes, night sweats, and sleep disturbances in women who completed the program.

\section{Materials and methods Study design}

The study was a parallel, randomized, open-label investigation of MR versus AR conducted over 12 weeks in perimenopausal and postmenopausal women. The investigation was conducted at the Menopausal Clinic of Mahasarakham Provincial Hospital in northeastern Thailand from July 2011 to January 2012. The Mahasarakham Provincial Hospital ethics committee approved the study and all participants gave written informed consent.

\section{Participants}

We recruited 105 perimenopausal and postmenopausal women who visited the menopause clinic at Mahasarakham Provincial Hospital in northeastern Thailand. Of these, 88 women experiencing hot flashes, night sweats, or sleep disturbances were potentially eligible, and 71 (81\%) chose to participate. Figure 1 shows the reasons given for not participating in the study.

The inclusion criteria were age $45-65$ years and irregular menstruation within the previous 12 months or menopause (either surgical or spontaneous). Women who received any hormone therapy in the 3 months before the study and those who had uncontrolled hypertension ( $>95 \mathrm{mmHg}$ diastolic pressure) or used sedatives, tranquilizers, or antidepressant medication on a daily basis were excluded. Women visiting the clinic were questioned about their experience of hot flashes, night sweats, or sleep disturbances. Potential participants with menopausal symptoms underwent a medical examination by an attending gynecologist at the menopause clinic. Participants rated their symptoms subjectively using a severity rating scale, and those with a severity score $\geq 5$ were invited to participate in the study. Those who expressed an interest in relaxation training to reduce hot flashes were randomly assigned to either the AR group or the MR group. Participants were given the option to discontinue their treatment at any time without jeopardizing their future care.

\section{Severity rating scale}

The severity rating scale was created by one of the investigators and validated in 10 menopausal women. Using this 


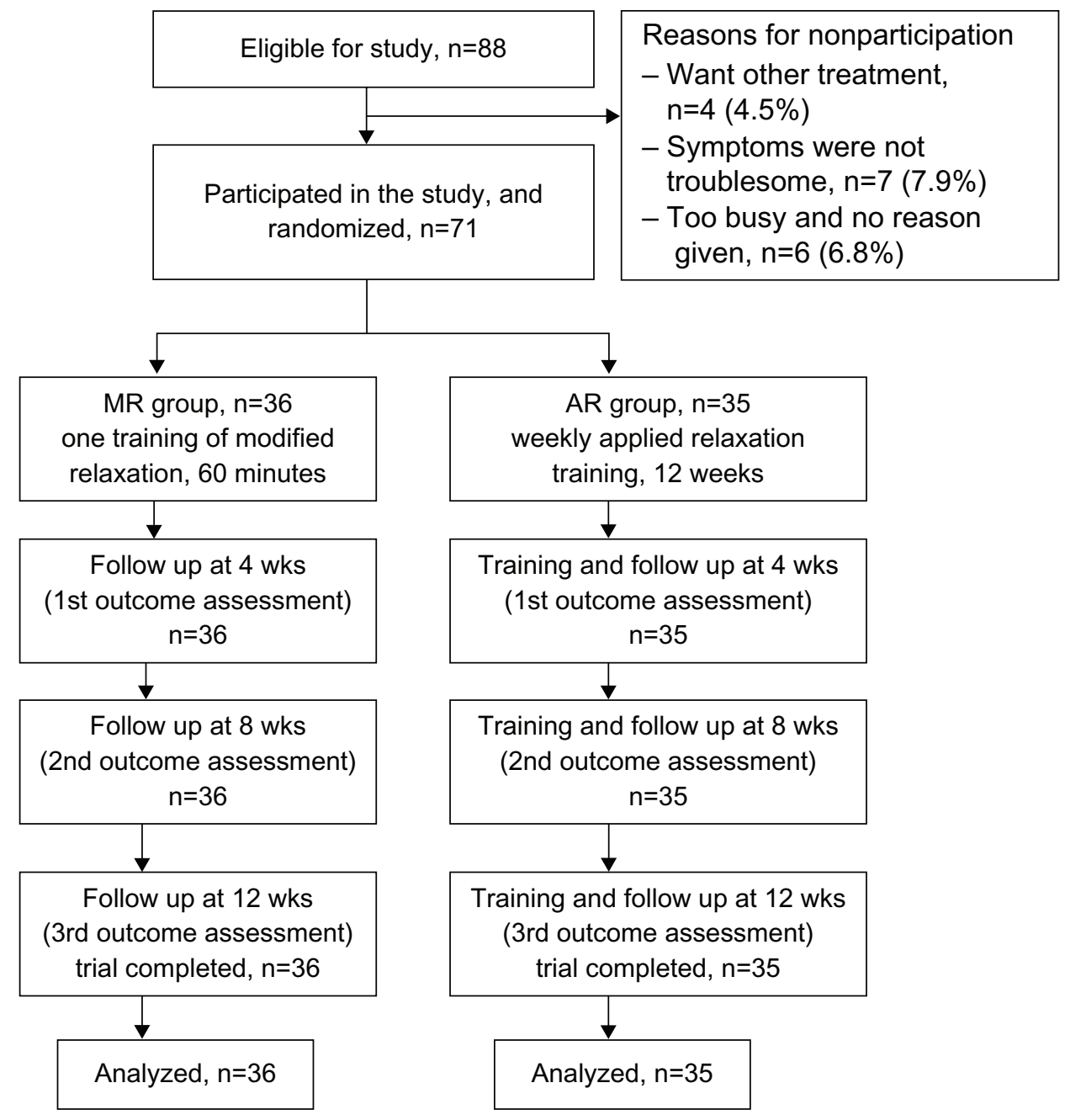

Figure I Patient recruitment and follow up.

Abbreviations: $M R$, modified version of $A R ; A R$, applied relaxation; wks, weeks.

instrument, the severity of hot flashes, night sweats, and sleep disturbances are subjectively rated using a five-point scale from 0 ("not at all") to 4 ("extremely"). Items in scale consists of three menopausal symptoms as hot flashes, night sweats, and sleep disturbances. The total severity score ranges from 0 to 12 .

\section{Intervention procedures}

Participants received relaxation training from two professional physical therapists under the supervision and care of their attending gynecologists. The physical therapists met before the start of the study to develop and standardize the scope and content of their teaching. One physical therapist was responsible for the AR training and the other supervised the MR training throughout the study. The trainer and participants met in small groups of 4-6 people.

Participants in the AR group attended 12 weekly sessions lasting 60 minutes each. Information about menopause, theories about hot flashes, and the rationale behind the use of AR as a coping technique for handling sudden, unanticipated symptoms was discussed during the first session. The first part of the progressive relaxation program (contraction and relaxation of muscles in the arms, face, neck, and shoulders) was taught following this discussion. In session 2, a program of progressive relaxation of the whole body was taught to participants. Session 3 provided a condensed version of progressive relaxation (relaxation without muscle straining). Cue-controlled relaxation was introduced (self-instructed relaxation) and practiced in session 4. Sessions 5 and 6 focused on differential relaxation (relaxation of non-used muscle groups), and sessions 7 and 8 focused on rapid relaxation. After completing session 8, participants were able to relax within 20-30 seconds. Application of the training began in sessions 9 and 10, in which participants were asked to use rapid relaxation as soon as their vasomotor symptoms 
appeared. Sessions 11 and 12 were used for rehearsal and a summary of the maintenance program. Participants were given a handout on AR after each session, and were asked to practice each component at home at least once a day. There were 12 total staff times and 12 telephone calls in this group.

Participants in the MR group received training from their physical therapist in one 60-minute session at the beginning of the study. The content of the MR technique has been validated in a study of 10 menopausal women, with a scale reliability coefficient of 0.76 .

The physical therapist was in contact by telephone with each participant for 12 consecutive weeks and answered any questions relevant to the technique or education, encouraged practice, and addressed general well being. This was substituted for the once-weekly class to maintain a like amount of communication with participants. The session consisted of four parts. The first addressed menopausal symptoms, to enhance understanding among the participants and teach awareness of tension in a particular area of the body. In the second part, participants were encouraged to maximize tension in the affected area, followed by stretching and release of the tension. In the third part, participants were asked to focus their attention on a particular area of the body, to recognize relaxation, and practice relaxing that area. In the final part of the session, participants were asked to commit to practicing at home and were given a summary of the maintenance program. Participants were given a handout on MR after the training session. They were also asked to practice MR at home once a day for 15-20 minutes during their leisure time at least 5 days a week for the following 12 weeks.

\section{Monitoring}

Participants in both groups kept specific daily records of the frequency and severity of their menopausal symptoms in a logbook. They were also asked to check the completeness of the records once a week. The logbooks were collected when the subjects visited the menopause clinic at 4, 8 , and 12 weeks after intervention. Participants also completed the severity rating scale and reported the frequency of symptoms at these visits. The research nurse determined completeness of the data, and all data were copied into a standardized medical record. Most participants (67, 94.4\%) adhered to the protocol. The trainers contacted participants by telephone once a week to answer questions, resolve any problems, assess symptoms, and monitor AR/MR practice. One physical therapist was responsible for telephone calls in the AR group and another was responsible for telephone calls to the MR group throughout the study.

\section{Statistical analyses \\ Randomization}

The randomization sequence was created using Stata 10 (StataCorp LP, College Station, TX, USA) statistical software and was center-stratified with a 1:1 allocation using a computer-generated random block size of 4 and 8 . The allocation sequence was placed in sequentially numbered, opaque, sealed, and stapled envelopes and concealed from the researcher enrolling and assessing participants.

\section{Outcome variables}

The outcome variables included change in severity rating scale score and change in frequency of hot flashes, night sweats, and sleep disturbances. Statistical analyses were performed using Stata software version 10 (StataCorp LP). The analysis was conducted as "intention-to-treat," with all participants included in the analysis. Descriptive statistics were applied to the baseline demographics, clinical characteristics, and dichotomous and continuous data. Chi-square $\left(\chi^{2}\right)$ and Student's $t$-tests were used to assess differences between groups. The Wilcoxon rank-sum test assessed differences in the rating scale variables. A $P$-value of 0.05 (two-tailed) was set for statistical significance.

\section{Sample size}

The sample size was based on a pilot study of 20 participants, comprising eleven in the AR group and nine in the MR group. Mean improvement in Menopausal Rating Scale (MRS) severity score after 4 weeks of treatment was $10.68 \pm 3.5$ in the AR group and $15.33 \pm 3.11$ in the MR group. Three subjects (28\%) in the AR group dropped out before the end of the pilot study, while all subjects (100\%) in the MR group completed the program. Using an alpha error of 0.05 (two-sided) and a power of $90 \%$, the required sample size per group was five. Since this was a small number, we increased the sample size in each arm to a minimum of 30 to provide a normal acceptable approximation. ${ }^{20}$

\section{Results}

The age range of the participants was $45-60$ years. The mean age of the groups was significantly different $(P<0.01)$. There were no significant differences in mean age at perimenopause, mean age at post menopause, body mass index, or menopausal status. All participants were nonsmokers and did not consume alcoholic beverages, and all completed the study (Table 1). 
Table I Demographic characteristics of participants

\begin{tabular}{|c|c|c|c|}
\hline \multirow[t]{2}{*}{ Characteristics } & \multirow{2}{*}{$\begin{array}{l}\text { MR group } \\
(n=36) \\
\text { Mean (SD) }\end{array}$} & \multirow{2}{*}{$\begin{array}{l}\begin{array}{l}\text { AR group } \\
(n=35)\end{array} \\
\text { Mean }(S D)\end{array}$} & \multirow{2}{*}{$\begin{array}{l}\text { Statistical } \\
\text { test and } \\
P \text {-value }\end{array}$} \\
\hline & & & \\
\hline Age, years & $49.8(3.8)$ & $52.5(5.1)$ & $0.0 \mathrm{I}^{t}$ \\
\hline Age at perimenopause, years & $45.9(6.0)$ & 46.9 (3.9) & $0.62^{t}$ \\
\hline Age at postmenopause & $47.4(0.6)$ & $48.9(0.8)$ & $0.18^{t}$ \\
\hline BMI $\left(\mathrm{kg} / \mathrm{m}^{2}\right)$ & $25.6(3.8)$ & $25.2(4.2)$ & $0.15^{t}$ \\
\hline Menopausal status & & & $0.18^{x^{2}}$ \\
\hline - Perimenopausal, n (\%) & $18(25.4)$ & $12(16.9)$ & \\
\hline - Postmenopausal, n (\%) & $18(25.4)$ & $23(32.4)$ & \\
\hline \multicolumn{4}{|l|}{ Severity score at baseline } \\
\hline Total severity & $6.5(4.5)$ & $5.0(2.0)$ & $0.92^{t}$ \\
\hline Hot flashes & $2.0(1.0)$ & $2.0(1.0)$ & $0.86^{t}$ \\
\hline Night sweats & $2.0(1.0)$ & $2.0(1.0)$ & $0.69^{t}$ \\
\hline Sleep disturbances & $2.0(2.5)$ & $2.0(1.0)$ & $0.82^{t}$ \\
\hline
\end{tabular}

Notes: 'Student's t-test; $x^{2} \mathrm{Chi}$-square test. Maximum range of total severity score at baseline and 12 weeks in MR group $=1 \mathrm{I}$ and 3, in $A R$ group $=10$ and 3. Maximum range of hot flashes severity score at baseline and 12 weeks in $M R$ group $=4$ and 2 , in $A R$ group $=3$ and 2. Maximum range of night sweats severity score at baseline and $I 2$ weeks in $M R$ group $=4$ and $I$, and in $A R$ group $=3$ and 0 . Maximum range of sleep disturbance severity score at baseline and 12 weeks in MR group $=4$ and I, and in $A R$ group $=4$ and $I$.

Abbreviations: AR, applied relaxation; BMI, body mass index; MR, modified version of $A R ; S D$, standard deviation.

\section{Comparison between MR and AR}

We tested the effectiveness of relaxation by comparing the two groups regarding changes in the severity rating scores for menopausal symptoms over time. There were decreases in total severity scores for hot flashes, night sweats, and sleep disturbances in both groups after 12 weeks $(z=-0.09, P=0.93$, Table 2). The severity score for hot flashes in the MR group was more decreased than in the AR group $(z=-2.33, P=0.02)$. The severity of both night sweats and sleep disturbances decreased in both groups, but the changes were not statistically significant. The frequency of hot flashes, night sweats, and sleep disturbances was decreased in both groups but there were no statistically significant differences (Table 2).

\section{Discussion}

This prospective randomized study shows that total severity score improved in both treatment groups from baseline to the end of the 12-week treatment period. We also observed dramatic decreases in the outcomes of hot flashes, night sweats, and sleep disturbances.

Although the MR technique is a very brief training program, the present findings show equal effectiveness of the MR and AR techniques (decreased severity score in both groups). This result is similar to that of two previous studies reporting the AR technique to be effective in reducing hot flashes. ${ }^{21-23}$

While the present study was a randomized, open-label, intention-to-treat analysis, it does have some limitations. First, the sample size might have been too small to detect a benefit in reducing menopausal symptoms. Second, although the participants were assigned randomly to the intervention groups, the open-label design can still cause bias toward the study treatments. Third, the study duration of 12 weeks might have been too short to determine the effects of MR and AR in individuals with menopausal symptoms. Although a trend toward reduction in frequency and severity was observed, the treatment effect may not be sustained after 12 weeks. ${ }^{20,22,23}$ This may be due to the study design. The MR intervention was a more intensive training program, which could extend the benefits beyond the learning phase. Fourth, we included women for whom menstruation had ceased for only a year or more. These women often experience particularly severe vasomotor symptoms and sleep disturbances. However, there was a risk that menstruation would return in some of these women, and this happened in two cases, both in the AR group. It is possible that these cases weakened the outcome of the study due to inconsistency of their symptoms. Fifth, the

Table 2 Change in severity and frequency of hot flashes, night sweats, and sleep disturbances

\begin{tabular}{|c|c|c|c|c|c|}
\hline \multirow[t]{2}{*}{ Variable } & \multicolumn{2}{|c|}{ MR group $(n=36)$} & \multicolumn{2}{|c|}{ AR group $(n=35)$} & \multirow[t]{2}{*}{ z, P-value } \\
\hline & Baseline & 12 weeks & Baseline & I 2 weeks & \\
\hline Total severity & $6.5(4.5)$ & $-5.0(3.5)$ & $5.0(2.0)$ & $-5.0(2.0)$ & $z=-0.09, P=0.93$ \\
\hline \multicolumn{6}{|l|}{ Severity by symptoms } \\
\hline Hot flashes & $2.0(1.0)$ & $-2.0(1.0)$ & $2.0(1.0)$ & $-1.0(2.0)$ & $z=-2.33, P>0.02 *$ \\
\hline Night sweats & $2.0(1.0)$ & $-2.0(1.0)$ & $2.0(1.0)$ & $-2.0(1.0)$ & $z=-0.67, P=0.5 \mathrm{I}$ \\
\hline Sleep disturbance & $2.0(2.5)$ & $-2.0(2.0)$ & $2.0(1.0)$ & $-2.0(3.0)$ & $z=0.34, P=0.74$ \\
\hline \multicolumn{6}{|l|}{ Frequency by symptoms } \\
\hline Hot flashes/day & $2.0(2.0)$ & $-1.0(1.0)$ & $2.0(2.0)$ & $-1.0(1.0)$ & $z=-0.55, P=0.58$ \\
\hline Night sweats/day & $2.0(2.0)$ & $-2.0(2.0)$ & $2.0(1.0)$ & $-2.0(1.0)$ & $z=-0.60, P=0.55$ \\
\hline Sleep disturbance/week & $2.0(3.0)$ & $-2.0(3.0)$ & $2.0(3.0)$ & $-2.0(3.0)$ & $z=-0.19, P=0.85$ \\
\hline
\end{tabular}

Notes: Severity score expressed as median (interquartile range); *Wilcoxon rank-sum test, $P<0.05$.

Abbreviations: $A R$, applied relaxation; MR, modified version of $A R$. 
severity scale for measuring the menopausal symptoms was relatively specific. Other methods could have been used to evaluate the effects of therapy. For example, a visual analog scale ${ }^{24}$ can be used as a measure of menopausal symptoms because it is a simple technique for measuring subjective experiences. However, studies using the visual analog scale have not found improvements in night sweats and sleep disturbance symptoms. Accordingly, we used a nonstandard instrument to assess menopausal symptom severity and this may limit the generalizability of the results. Sixth, the study assessed only a few types of menopausal symptoms (ie, vasomotor symptoms). Although this limitation was expected at the inception of the study, to err on the side of caution we only assessed vasomotor symptoms (hot flashes and night sweats) and sleep disturbances. The generalization of these findings to perimenopausal and postmenopausal women with other symptoms (cognitive and sexual problems) may not be possible. Seventh, there was no monitoring of adherence to treatment. We could not determine the duration and frequency of MR practice or any lifestyle changes in the intervention and control groups. Last, other benefits of MR, for example, enhanced quality of life, were not assessed.

Our findings are in contrast with those reported by Freedman and Woodward, ${ }^{25}$ who found no reduction in hot flashes with muscle relaxation, but are consistent with the findings of Hunter and $\mathrm{Liao}^{26}$ who found that hot flashes were reduced using behavioral therapy techniques.

This study also has several strengths. First, the recruitment interview assessed women's experience of menopausal symptoms and included a discussion of management strategies. The patient-therapist relationship was maintained by weekly telephone calls throughout the trial. We feel that the benefits obtained by the participants were achieved as a result of this frequent contact. We also believe that participants should be advised to practice regularly and that more follow-ups should be included in the program to maintain the therapeutic effects. Second, although self-reported measures of vasomotor symptoms and sleep disturbances have been shown to correspond well with other methods of measurement, the use of diaries may introduce bias. However, relaxation training is a behavioral modification, and writing down the number of practice sessions in a diary may be helpful for consistency. We suggest that the availability of a variety of behavior modification-based techniques for relieving symptoms associated with menopause will allow women to choose a therapy that meets their individual needs, and this increases the probability of selecting the most effective response from among these behavioral therapy techniques. ${ }^{27}$

Since MR and AR techniques had equal success in relieving menopausal symptoms, MR techniques could complement or be used as an alternative to AR. The use of relaxation training reduced both the frequency and severity of menopausal symptoms, and this effect may have been related to practice after the training sessions. Although the mean frequency score reduction and median score severity appear to be small, this may represent a real benefit for some women. We suggest that both MR and AR be made available so that women may make a personal choice. However, we believe that the relationship between MR, AR, and menopausal symptoms should be evaluated further.

\section{Conclusion}

The total severity score, as well as frequency and severity scores for hot flashes, night sweats, and sleep disturbances tended to decrease in perimenopausal and postmenopausal women in the MR and AR groups. Studies with large sample sizes are necessary to clarify or confirm our results.

\section{Acknowledgment}

The authors acknowledge the physiotherapists and professional nurses at Mahasarakham Provincial Hospital who provided the relaxation training and collected the data for this study.

\section{Disclosure}

The authors report no conflicts of interest in this work.

\section{References}

1. McKinlay MS, Brambilla JD, Posner J. The normal menopause transition. Maturitas. 1992;14(2):103-115.

2. Freeman EW, Sammel MD, Lin H, et al. Symptoms associated with menopausal transition and reproductive hormones in midlife women. Obstet Gynecol. 2007;110(2 Pt 1):230-240.

3. Kaufert AP, Gilbert P. The Manitoba Project: a re-examination of the link between menopause and depression. Maturitas. 1992;14(2):143-155.

4. Dennerstein L, Smith MA, Morse AC, et al. Menopausal symptoms in Australian women. Med J Aust. 1993;159(4):232-236.

5. Lopez FJ, Finn PD, Lawson MA, Negro-Vilar A. Regulation of the hypothalamic-pituitary-gonadal axis: role of gonadal steroids and implications for the menopause. In: Lobo RA, Kelsey J, editors. Menopause: Biology and Pathobiology. San Diego, CA: Academic Press; 2000.

6. Dennerstein L, Dudley E, Hopper J, Guthrie J, Burger H. A prospective population-based study of menopausal symptoms. Obstet Gynecol. 2000;96(3):351-358.

7. Gold BE, Sternfeld B. Relation of demographic and lifestyle factors to symptoms in a multi-racial/ethnic population of women $40-55$ years of age. Am J Epidemiol. 2000;152(5):463-473.

8. Col NF, Guthrie JR, Politi M, Dennerstein L. Duration of vasomotor symptoms in middle-aged women: a longitudinal study. Menopause. 2009; $16: 453-457$. 
9. Soules MR, Sherman S, Parrott E, et al. Executive summary: stages of reproductive aging workshop (STRAW) Park City, Utah, Jul 2001. Menopause. 2001;8(6):402-407.

10. Hickey M, Davis SR, Sturdee DW. Treatment of menopausal symptoms: what shall we do now? Lancet. 2005;366(9483):409-421.

11. Rossouw JE, Anderson GL, Prentice RL, et al. Risks and benefits of estrogen plus progestin in healthy postmenopausal women: principal results from the Women's Health Initiative Randomized Control Trial JAMA. 2002;288(3):321-333.

12. North American Menopause Society. Treatment of menopauseassociated vasomotor symptoms: position statement of The North American Menopause Society. Menopause. 2004;11(1):11-33.

13. Newton K, Buist DS, Keenan NL, Anderson LA, LaCroix AZ. Use of alternative therapies for menopause symptoms: results of a population based survey. Obstet Gynecol. 2002;100(1):18-25.

14. Blair YA, Gold EB, Azari RA, et al. Use of conventional and complimentary health care during the transition to menopause: longitudinal results from the Study of Women's Health Across the Nation (SWAN). Menopause. 2005;12(1):31-39.

15. Daley AJ, MacArthur C, McManus R, et al. Factors associated with the use of complementary medicine and non-pharmacological interventions in symptomatic menopausal women. Climacteric. 2006;9(5): 336-346.

16. Hoffman WJ, Benson H, Arns AP. Reduced sympathetic nervous system responsivity associated with the relaxation response. Science. 1981;215(4529):190-192.

17. Nedstrand E, Wijma K, Wyon Y, Hammar M. Applied relaxation and oral estradiol treatment of vasomotor symptoms in postmenopausal women. Maturitas. 2005;51(2):154-162.
18. Freedman RR, Woodward S, Brown B, Javaid IJ, Pandey GN. Biochemical and thermoregulatory effects of behavioral treatment for menopausal hot flushes. Menopause. 1995;2(4):211-218.

19. Innes KE, Selfe TK, Vishnu A. Mind-body therapies for menopausal symptoms: a systematic review. Maturitas. 2010;66(2):135-149.

20. Lachin JM. Introduction to sample size determination and power analysis for clinical trials. Control Clin Trials. 1981;2(2):93-113.

21. Germaine LM, Freedman RR. Behavioral treatment of menopausal hot flashes: evaluation by objective methods. J Consult Clin Psychol. 1984;52(6):1072-1079.

22. Nedstrand E, Wijma K, Wyon Y, Hammar M. Vasomotor symptoms decrease in women with breast cancer randomized to treatment with applied relaxation or electro-acupuncture: a preliminary study. Climacteric. 2005;8(3):243-250.

23. Zaborowska E, Brynhildsen J, Damberg S, et al. Effects of acupuncture, applied relaxation, estrogens and placebo on hot flush in postmenopausal women: an analysis of prospective, parallel randomized studied. Climacteric. 2007;10(1):38-45.

24. McCormack HM, Horne DJ, Sheather S. Clinical applications of visual analogue scales: a critical review. Psychol Med. 1988;18(4): 1007-1019.

25. Freedman RR, Woodward S. Behavioral treatment of menopausal hot flushes: evaluation by ambulatory monitoring. Am J Obstet Gynecol. 1992;167(2):436-439.

26. Hunter M, Liao KLM. Evaluation of four session cognitive behavioral intervention for menopausal hot flushes. Br J Health Psychol. 1996;1: 113-125.

27. D'Zurilla, Goldfried TJ, Marvin R. Problem solving and behavior modification. J Abnorm Psychol. 1971;78(1):107-126.
International Journal of Women's Health

\section{Publish your work in this journal}

The International Journal of Women's Health is an international, peerreviewed open-access journal publishing original research, reports, editorials, reviews and commentaries on all aspects of women's healthcare including gynecology, obstetrics, and breast cancer. The manuscript management system is completely online and includes

\section{Dovepress}

a very quick and fair peer-review system, which is all easy to use. Visit http://www.dovepress.com/testimonials.php to read real quotes from published authors. 